\title{
Características Psicométricas y Estructura Factorial del School Burnout Inventory Student (SBI-U-9) en Estudiantes Universitarios Mexicanos
}

\author{
Psychometric Characteristics and Factor Structure of the School Burnout Inventory \\ Student (SBI-U-9) in Mexican University Students
}

\author{
Maricela Osorio Guzmán ${ }^{1}$, Carlos Prado Romero², Santa Parrello ${ }^{3}$ y Georgina Eugenia Bazán Riverón ${ }^{4}$
}

\begin{abstract}
Resumen
El burnout académico se define como una respuesta emocional negativa, persistente, relacionada con el desempeño académico, formada por una sensación de encontrarse exhausto, una actitud cínica o de distanciamiento con el significado y la utilidad de los estudios y un sentimiento de incompetencia académica como estudiante; este fenómeno tiene importantes efectos en la salud de quienes lo padecen por lo que se torna imperativo adaptar instrumentos que permitan su detección en poblaciones específicas. Se evaluó a 578 estudiantes universitarios mexicanos mediante el School Burnout Inventory Student (SBI-U-9) con el objetivo de analizar sus características psicométricas, estructura factorial e invarianza entre hombres y mujeres. Los resultados muestran que el SBI-U-9 presenta índices de ajuste adecuados. Se concluye que el instrumento posee características psicométricas adecuadas para su aplicación en estudiantes universitarios mexicanos, y se confirma el constructo trifactorial del burnout académico en la muestra.
\end{abstract}

Palabras clave: enfermedad profesional, estudiantes universitarios, malestar de la juventud, psicometría

\begin{abstract}
Academic burnout is defined as a negative and persistent emotional response, related to academic performance, formed by a feeling of being exhausted, a cynical or estrangement attitude with the meaning and usefulness of studies and a feeling of academic incompetence as a student. This phenomenon has important effects on the health of those who suffer from it, so it is imperative to adapt instruments that allow the detection in specific population. 578 Mexican college students were evaluated through the School Burnout Inventory Student (SBI-U-9) with the objective of analyzing their psychometric characteristics, factor structure and invariance between male and female. The results show that the SBI-U-9 has fit index adequate. It is concluded that the instrument has adequate psychometric characteristics for its application in Mexican college students, and the trifactorial construct of academic burnout in the sample is confirmed.
\end{abstract}

Keywords: professional illness, college students, youth discomfort, psychometry

\footnotetext{
${ }^{1}$ Doctora en Ciencias Psicológicas y Pedagógicas: Psicología de la Salud, Prevención del Riesgo Individual y Social. Profesora Titular C, Tiempo Completo Definitiva. Universidad Nacional Autónoma de México, Facultad de Estudios Superiores Iztacala. Av. De los Barrios 1, Los Reyes Iztacala, Tlalnepantla Estado de México, México CP 54090. Tel.: (55) 56231333 ext.39806. Correo: mosorio@unam.mx

${ }^{2}$ Licenciado en Psicología y Doctorando en Psicología. Universidad Nacional Autónoma de México, Facultad de Estudios Superiores Iztacala. Av. De los Barrios 1, Los Reyes Iztacala, Tlalnepantla Estado de México, México CP 54090. Tel.: (55)56231333 ext.39806. Correo: carlosprador9318@gmail.com

${ }^{3}$ Doctorado en Metodología de las Ciencias Relacionales y de la Comunicación. Ricercatrice e Docente di Psicologia dello Sviluppo e dell'Educazione, Dipartimento di Studi Umanistici. Università degli Studi di Napoli Federico II via Porta di Massa 1 - 80138 Napoli, Italia. Tel.: 0812535449. Correo: parrello@unina.it

${ }^{4}$ Doctorado en Psicopatología Infantil. Profesora Titular B, Tiempo Completo Definitiva. Universidad Nacional Autónoma de México, Facultad de Estudios Superiores Iztacala. Av. De los Barrios 1, Los Reyes Iztacala, Tlalnepantla Estado de México, México CP 54090. Tel.: (55)56231333 ext.39806. Correo: gebrmx@yahoo.com.mx
} 


\section{Introducción}

El síndrome de burnout, también conocido como síndrome de estar quemado, síndrome de desmoralización o síndrome de agotamiento emocional o profesional, es considerado como una problemática laboral que repercute en la conducta humana, provoca afectaciones en la salud física y psicológica de los individuos (Balsera, et al., 2016; Feitosa, Sousa, Alves, Gómez, \& Méndez, 2016; Weber, \& Jaekel, 2000), en el rendimiento laboral y académico; y potencialmente puede provocar problemas de tipo personal, familiar y social (De la Rosa, et al., 2015).

El término, fue utilizado por primera vez en 1970 por Freudenberger, para hacer referencia al estrés provocado en el cuidado y atención profesional brindado por organizaciones de salud y en los trabajadores que atienden a otras personas (Boada, Merino, Sánchez, Prizmic, \& Vigil, 2015; Pedrosa, \& García, 2016). A su vez, las investigaciones iniciales del síndrome se centraron originalmente en el estudio de profesiones asistenciales (Freudemberger, 1974, Maslach, \& Jackson 1982), por lo que éste síndrome era conceptualizado como efecto de la interacción con los usuarios de este tipo de servicios. El término inicialmente se definía como un estrés crónico producido por el contacto con los clientes, que lleva a la extenuación, al distanciamiento emocional y a dudas acerca de la capacidad para realizar efectivamente una labor (Maslach, 1976). En función de este enfoque, se elaboró el MBI (Maslach Burnout Inventory) de Maslach y Jackson, en 1981 (Moreno, Rodríguez, \& Escobar, 2001); el cual esencialmente consistió en una operacionalización instrumental del constructo (Maslach, \& Schaufeli, 1993). El instrumento resultó de tanto interés que pronto se planteó la reelaboración teórica y operacional del burnout aplicado a profesiones no asistenciales, surgiendo así el MBI General Survey (MBI-GS) enfocado a evaluar las actitudes ante el propio trabajo y el burnout (Maslach, Jackson, \& Leiter, 1996). Posteriormente Schaufeli, Salanova, González-Romá y Bakker (2002), operacionalizaron el burnout al contexto académico, adaptando el MBI-GS a estudiantes universitarios, lo cual dio origen al cuestionario MBI-SS (Maslach Burnout Inventory-Student
Survey). Para hacerlo, se partió de la presunción de que la actividad académica del estudiante es equivalente a la de un trabajador formal (Hederich, \& Caballero, 2016). La aplicación del MBI-SS demostró que, al igual que los trabajadores, los estudiantes pueden reflejar agotamiento por las demandas del estudio (agotamiento), además de actitudes de desinterés, autosabotaje frente a las actividades académicas y dudas acerca del valor del estudio (cinismo), así como un sentimiento de incompetencia como estudiantes (autoeficacia negativa).

Otra línea de investigación más reciente es la dirigida por Salmela-Aro, Kiuru, Leskinen, y Nurmi, (2009), quienes diseñaron y validaron un nuevo instrumento, de nueve ítems, para la determinación de burnout académico: el SchoolBurnout Inventory (SBI-U-9), este instrumento conserva las tres escalas destinadas a evaluar las diferentes dimensiones del constructo (agotamiento emocional y físico, actitud cínica de distanciamiento mental hacia las actividades académicas, sentimientos de inadecuación al trabajo y pérdida de confianza en las propias capacidades profesionales), y originalmente fue diseñado en inglés (Hederich, \& Caballero, 2016); aunque ha sido traducido y aplicado en Finlandia (Salmela-Aro, \& Kunttu, 2010), Colombia (Caballero, Hederich, \& Palacio, 2010; Caballero, 2012; Palacio, Caballero, González, Gravini, \& Contreras, 2012; Aguilar-Bustamante, \& RiañoHernández, 2013), España (Moyano, \& RiañoHernández, 2013; Boada, et al., 2015), Italia (Fiorilli, Galimberti, De Stasio, Di Chiacchio, \& Albanese, 2014; Parrello, Ambrosetti, Iorio, \& Castelli, 2019), Perú (Merino, Delgadillo, \& Caballero, 2013) y México (Ochoa, Musi, Olivas \& Espinosa, 2018). De acuerdo a Montiel y Chong (2019), todos los resultados publicados hasta el momento muestran una estructura trifactorial prácticamente idéntica a la presentada por el MBI-SS, así como niveles de fiabilidad aceptables para cada escala (Charry, Garzón, Pozo $\&$ Bretones, 2018).

Por otra parte, el trabajo académico a nivel superior, desde un punto de vista psicológico y social, es comparable a las actividades que lleva a cabo un empleado, entre otras cosas porque como cualquier asalariado, forman parte de una organización donde desempeñan un determinado 
rol, realizan tareas que requieren esfuerzo, tienen objetivos y/o metas que cumplir, y su rendimiento es constantemente evaluado y recompensado por sus profesores, quienes ejercen una función análoga a la de un supervisor en el ámbito laboral (Caballero, Abello, Raymundo, \& Palacios, 2007; Caballero, Bresó, \& González, 2015; Herrera, Mohamed, \& Cepero, 2016; Oliva, Santos, Abreu, Melo, \& Andrade, 2012; Dominguez \& Merino, 2019).

Además, es común que los estudiantes universitarios se enfrenten a estresores cotidianos como: largas horas de clase, muchas horas de estudio, realización de proyectos y tareas, relaciones conflictivas con sus profesores, desvalorización de sus capacidades por parte de los docentes, entre otros (Caballero, Bresó, \& González, 2015; Rosales \& Rosales, 2013); asimismo, tienen que superar desacuerdos con los compañeros y/o poco apoyo por parte de los mismos o de la propia familia, están expuestos a enfrentarse con desafíos, como horas muertas, dominio de otro idioma, sobrecarga académica, trabajos empalmados y poca disponibilidad de tiempo para estudiar entre examen y examen (Barradas, Trujillo, Sánchez \& López, 2017; Osorio, Parrello, \& Prado, en prensa).

Partiendo de lo anterior, este síndrome ha sido conceptualizado como burnout académico, y definido como una respuesta emocional negativa, persistente, relacionada con los estudios (Schaufeli, Salanova, González-Roma, \& Bakker, 2002), la cual está constituida por una sensación de encontrarse exhausto, de no poder más en las tareas como estudiante (agotamiento); una actitud cínica o de distanciamiento con el significado y la utilidad de los estudios que se están realizando (cinismo); y un sentimiento de incompetencia académica como estudiante (inadecuación) (Martínez \& Marques, 2005).

Así mismo, en diversos estudios se han reportado efectos específicos del burnout clasificados en: 1) psicosomáticos: alteraciones cardiovasculares, fatiga crónica, cefaleas, alteraciones gastrointestinales, dolores abdominales y musculares, alteraciones respiratorias y del sueño, alteraciones dermatológicas y menstruales, así como disfunciones sexuales. 2) conductuales: abuso de drogas, fármacos y alcohol, ausentismo escolar, malas conductas alimentarias, incapacidad de relajarse. Y 3) emocionales: impaciencia, deseos de abandonar los estudios y/o el trabajo, irritabilidad, dificultad para concentrarse debido a la ansiedad experimentada, depresión, baja autoestima, falta de motivación (Rosales, \& Rosales, 2013; Caballero, Bresó, \& González, 2015).

Además, Caballero, Hederich y Palacio (2010: 136-137), reportaron variables asociadas a la presencia del burnout académico y las categorizan en: 1) variables del contexto académico; 2) variables relacionadas con el contexto ambiental y/o social; y 3) variables intrapersonales.

Particularmente en México, dos datos subrayan la relevancia social del estudio del burnout académico en universitarios; el primero, según la Organización Mundial de la Salud (OMS) México ocupa el primer lugar en estrés y escasa funcionalidad laboral y académica, puesto que el $75 \%$ de su población sufre el síndrome de burnout o fatiga por estrés laboral superando a países como China (73\%) y Estados Unidos (59\%); mientras que el segundo, según la Organización para la Cooperación y el Desarrollo Económico (OCDE) los niveles de estrés a los que se sienten sometidos los alumnos mexicanos para alcanzar sus metas académicas son de los más elevados de los países miembros, ya que el $50 \%$ se pone muy tenso a la hora de estudiar y el 79\% se muestra altamente preocupado por obtener malas calificaciones en la escuela (Montiel \& Chong, 2019: 75). Por otra parte, algunos autores, señalan que es necesario adaptar los instrumentos a cada uno de los países y muestras donde serán aplicados (Balluerka, Gorostiaga, Alonso, \& Haramburu, 2007), por lo que el objetivo de este trabajo fue analizar las características psicométricas, estructura factorial e invarianza entre hombres y mujeres del School Burnout Inventory (SBI-U-9) versión en castellano de Boada, et al. (2015), en una muestra de estudiantes universitarios mexicanos, debido a la relevancia social que comporta el estudio de este fenómeno y a que al momento de realizar este trabajo, no se encontró evidencia teórica de un estudio similar en México.

\section{Método}

\section{Participantes}

578 estudiantes de 49 carreras agrupadas en 4 áreas de conocimiento de la Universidad Nacional Autónoma de México (UNAM), cuya media de 
edad fue de 20.08 años (DS=4.1), siendo el $46.5 \%$ de género masculino y $53.5 \%$ del femenino; el $60.4 \%$ reside en la Ciudad de México y el resto en la zona conurbada, el semestre con mayor frecuencia fue el cuarto, la media del aprovechamiento general fue de 7.76/10 (DS=0.7). Respecto al área de conocimiento de los participantes, el $47.1 \%$ pertenece al área de ciencias biológicas y de la salud, el $26.1 \%$ al área de ciencias sociales, el $15.4 \%$ a humanidades y artes y finalmente el $11.3 \%$ al área de físico matemáticas. Todos aceptaron participar en la investigación y firmaron el consentimiento informado.

\section{Instrumentos}

Se aplicaron dos: cuestionario de datos sociodemográficos. Se elaboró un cuestionario para recabar información como edad, género, carrera, lugar de procedencia y promedio. School Burnout Inventary para estudiantes universitarios (SBI-U-9). Se aplicó el instrumento diseñado por Salmela-Aro, Kiuru, Leskinen y Nurmi (2009) en la versión validada al castellano por Boada et al. (2015), en estudiantes universitarios de España. Este instrumento está compuesto por 9 ítems en escala Likert que va de 1 (completamente en desacuerdo) a 6 (completamente de acuerdo) divididos en 3 escalas. La escala de "agotamiento" está formada por cuatro ítems $(1,4,7,9)$ con un $\alpha=.70$, la escala para "cinismo" posee tres ítems $(2,5,6), \alpha=.77$ y 2 para "inadecuación” dos ítems (3 y 8 ) con alfa de .71 (ver Anexo).

\section{Procedimiento}

Los instrumentos fueron aplicados en las instalaciones de la Universidad Nacional Autónoma de México (UNAM) en aulas de las diferentes facultades y en la biblioteca central de Ciudad Universitaria, se identificó a los estudiantes y se les solicitó participar en la investigación, se les invitó a firmar el consentimiento informado, y a contestar los instrumentos; posteriormente se seleccionaron aquellos contestados de forma completa y adecuada, y se procedió a la captura de datos y al análisis correspondiente.

\section{Análisis de datos}

Se realizó un análisis factorial confirmatorio, para determinar las características de ajuste del modelo teórico de los autores del instrumento. El método utilizado fue el de máxima verosimilitud robusto -debido a que es el análisis recomendado para variables moderadamente normales (skew $<2$ y kurtosis $<7$; Finney \& DiStefano, 2006) con bootstraping de 500 muestras-. Los estadísticos de bondad de ajuste reportados son: Chi cuadrado $\left(\mathrm{X}^{2}\right)$, grados de libertad $(\mathrm{gl})$, tasa de ajuste $\left(\mathrm{X}^{2} / \mathrm{gl}\right)$, índice de ajuste comparativo (CFI), índice Tucker-Lewis (TLI), raíz de la media cuadrática residual estandarizada (SRMR), error de aproximación residual de la raíz de la media cuadrática (RMSEA) con intervalo de confianza (IC) del $90 \%$. Como norma general, se establece un buen ajuste del modelo cuando: tasa $\mathrm{X}^{2} / \mathrm{gl} \leq 3$; TLI $>.90$; CFI $>.95$; SRMR $\leq 06$; RMSEA $\leq 06$ (Hu, \& Bentler, 1999).

Finalmente, para evaluar la invarianza de medición (configuración, métrica, escalar y estricta) se efectuaron los análisis factoriales confirmatorios multi-grupo (Vanderberg \& Lance, 2000; Caycho, 2017). La invarianza de configuración implica la no variabilidad de la estructura entre los grupos, calculándose libremente las cargas factoriales, interceptos, y residuales; la invarianza métrica restringe la estructura y cargas factoriales; la invarianza escalar agrega la restricción de los interceptos; por último, la invarianza estricta restringe además los valores residuales. Para evaluar las diferencias de los modelos se utilizaron los cambios en los índices de ajuste CFI y RMSEA; se asume una fuerte invarianza cuando los cambios en $\Delta \mathrm{CFI} \leq .01, \quad \Delta \mathrm{RMSEA} \leq .01 \quad$ y $\quad p \quad$ de $\quad \Delta \mathrm{X}^{2} \geq 0.05$ (Cheung \& Rensvold, 2002).

Los análisis de datos se apoyaron en los programas estadísticos Factor 10.10.01 (Ferrando \& Lorenzo-Seva, 2017), SPSS 25 y AMOS 21.

\section{Resultados}

Se realizó un AFC utilizando el método de estimación de máxima verosimilitud robusto con 500 remuestreos (Bootstraping) los valores de ajuste del modelo con la presente muestra y el modelo propuesto por Boada et al., (2015), se pueden observar en la siguiente Tabla (ver Tabla 1).

Como se puede observar en la Tabla 1, los índices de bondad de ajuste se muestran ligeramente mejor a los observados en la muestra original. 
Tabla 1. Comparación de los valores de ajuste del análisis factorial confirmatorio de la muestra de estudiantes universitarios mexicanos y la muestra española de Boada et al., (2015)

\begin{tabular}{lcccccccc}
\hline Modelo & $\mathrm{X}^{2}$ & $\mathrm{gl}$ & $\mathrm{X}^{2} / \mathrm{gl}$ & $\mathrm{CFI}$ & $\mathrm{TLI}$ & RMSEA & SRMR & IC 90\% \\
\hline $\begin{array}{l}\text { Muestra } \\
\begin{array}{l}\text { Mexicana } \\
\text { Boada et al. }\end{array}\end{array}$ & 33.901 & 24 & 1.41 & .98 & .95 & .08 & .02 & $.07-.09$ \\
$(2015)$ & 86.413 & 24 & 3.58 & .95 & .93 & .06 & .03 & $.047-.078$ \\
\hline
\end{tabular}

Nota $\mathrm{X}^{2}=$ Chi cuadrado, gl=grados de libertad, $\mathrm{X}^{2} / \mathrm{gl}=$ tasa de ajuste, $\mathrm{CFI}=$ índice de ajuste comparativo, TLI=índice Tucker-Lewis, SRMR=raíz de la media cuadrática residual estandarizada, RMSEA=error de aproximación residual de la raíz de la media cuadrática, IC 90\%=intervalo de confianza del $90 \%$.

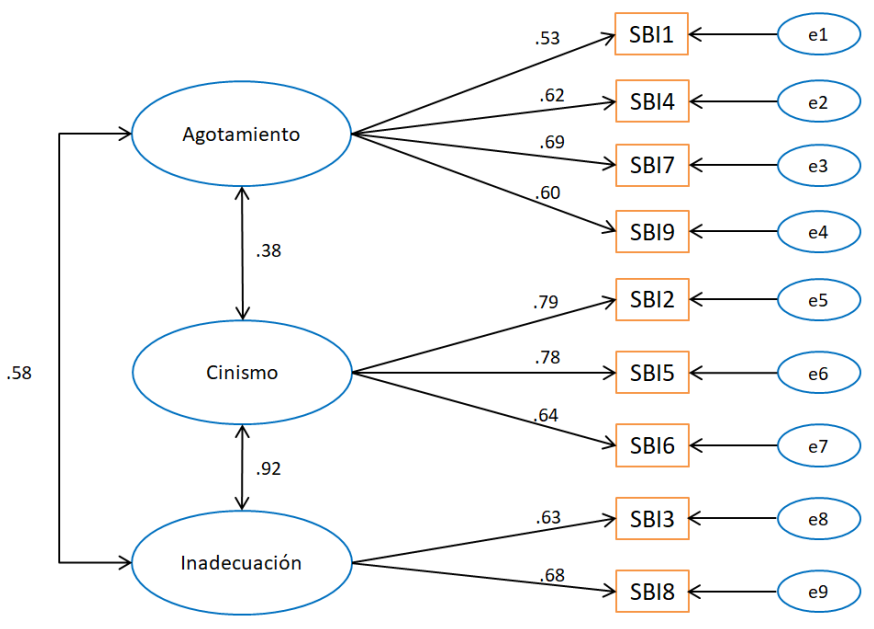

Modelo Boada y colaboradores (2015)

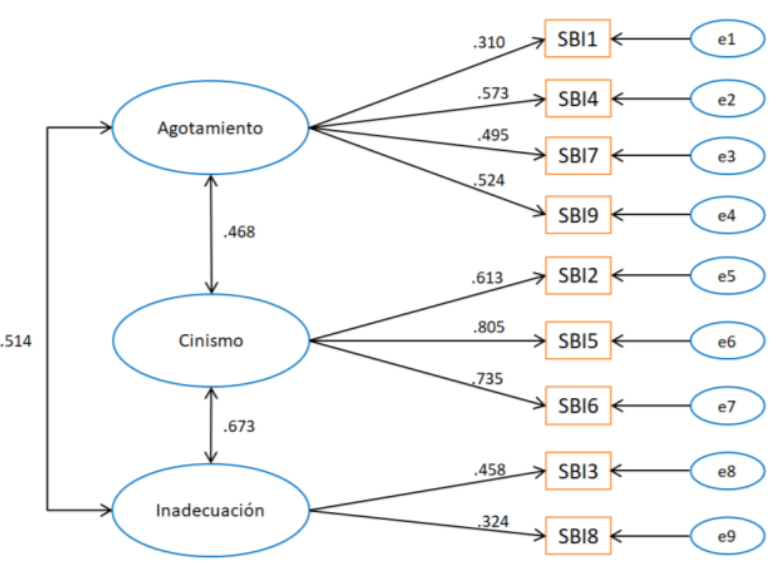

Modelo con muestra mexicana

Figura 1. Representación de la estructura factorial del modelo de Boada et al. (2015) y de la muestra mexicana

Tabla 2. Cargas factoriales estandarizadas en los AFC multi-grupo del modelo de invarianza de configuración

\begin{tabular}{lcc}
\hline \multirow{2}{*}{ Reactivo } & \multicolumn{2}{c}{ Cargas factoriales por sexo } \\
\cline { 2 - 3 } & Hombres & Mujeres \\
\hline SBI 1 & .546 & .529 \\
SBI 4 & .668 & .589 \\
SBI 7 & .302 & .510 \\
SBI 9 & .702 & .682 \\
SBI 2 & .710 & .733 \\
SBI 5 & .836 & .872 \\
SBI 6 & .663 & .751 \\
SBI 3 & .709 & .761 \\
SBI 8 & .610 & .714 \\
\hline
\end{tabular}

La representación gráfica de ambos modelos se puede apreciar a continuación (ver Figura 1).

Para evaluar la invarianza factorial del modelo entre hombres y mujeres se realizó un AFC multigrupo, en la Tabla 2 se observan los pesos factoriales de cada reactivo hacia su respectivo factor, todas las cargas factoriales fueron altas y significativas $(p<.01)$. Los índices de ajuste obtenidos mediante este análisis (CFI=.967; RMSEA $=.044 ; \mathrm{X}^{2} / \mathrm{gl}=2.133$ ) son adecuados (ver Tabla 2).
En la siguiente Tabla se observan los índices de ajuste de cada modelo de invarianza (configuración, métrica, escalar y estricta), así como el cambio y significación de cada modelo comparado.

Al realizar la comparación entre los modelos en la prueba de invarianza con el estadístico $\mathrm{X}^{2}$, se observa un valor de significación de la diferencia superior a $p=.05$, y cambios en el valor de los índices CFI y RMSEA inferiores a .01. Por lo tanto, se asume la invarianza factorial del modelo de evaluación del burnout académico entre hombres y mujeres (Elosua, 2005; Caycho, 2017; Charry, Garzón, Pozo, \& Bretones, 2018; Barrera, Navarro, \& Peris, 2015).

\section{Discusión}

Por la trascendencia social que conlleva el estudio del burnout en México y debido a que al momento de realizar la presente investigación solo se encontró un trabajo presentado en un congreso nacional, cuyo objetivo fue estimar la validez de contenido de la versión mexicana de SBI (12 ítems) 
Tabla 3. Índices de ajuste para las pruebas de los modelos de invarianza por sexo

\begin{tabular}{|c|c|c|c|c|c|c|c|c|}
\hline Modelo & $\mathrm{X}^{2}(\mathrm{gl})$ & $\mathrm{X}^{2} / \mathrm{gl}$ & CFI & $\begin{array}{l}\text { RMSEA } \\
\text { (IC 90\%) }\end{array}$ & Comp. & $\Delta X^{2}$ & $\Delta \mathrm{CFI}$ & $\triangle \mathrm{RMSEA}$ \\
\hline $\begin{array}{l}\text { 1-Invarianza de } \\
\text { configuración (Línea } \\
\text { base) }\end{array}$ & $\begin{array}{c}102.373 \\
(48)\end{array}$ & 2.133 & .967 & $\begin{array}{c}.044 \\
(.032-.056)\end{array}$ & & & & \\
\hline $\begin{array}{l}\text { 2-Invarianza métrica o } \\
\text { débil ( } \lambda \text { restringida) }\end{array}$ & $\begin{array}{l}109.526 \\
(54)\end{array}$ & 2.028 & .967 & $\begin{array}{c}.042 \\
(.031-.054)\end{array}$ & $2 \operatorname{con} 1$ & $\begin{array}{c}7.153(6) \\
p=.307\end{array}$ & $<.001$ & .002 \\
\hline $\begin{array}{l}\text { 3-Invarianza escalar o } \\
\text { fuerte }(\lambda \text { y } \tau \text { restringidos })\end{array}$ & $\begin{array}{l}124.939 \\
(63)\end{array}$ & 1.983 & .963 & $\begin{array}{c}.041 \\
(.031-.052)\end{array}$ & $3 \operatorname{con} 2$ & $\begin{array}{c}15.413(9) \\
p=.08\end{array}$ & .004 & .001 \\
\hline $\begin{array}{l}\text { 4-Invarianza estricta }(\lambda, \tau \\
\mathrm{y} \theta \text { restringidos) }\end{array}$ & $\begin{array}{c}148.233 \\
(78)\end{array}$ & 1.900 & .958 & $\begin{array}{c}.040 \\
(.030-.049) \\
\end{array}$ & $4 \operatorname{con} 3$ & $\begin{array}{c}23.294(15) \\
p=.08\end{array}$ & .005 & .001 \\
\hline
\end{tabular}

en muestras de adolescentes de 12 a 18 años del estado de Chihuahua México (Ochoa, Musi, Olivas, \& Espinosa, 2018); el objetivo del presente trabajo fue analizar las características psicométricas, estructura factorial e invarianza entre hombres y mujeres del SBI-U-9 de Boada, et al. (2015), en una muestra de estudiantes universitarios mexicanos. Atendiendo a los valores de los índices de bondad de ajuste se muestra que fueron adecuados de acuerdo al criterio presentado por diferentes autores (Hu \& Bentler, 1999; Manzano y Zamora, 2009; Furr, 2017), por lo que se presenta evidencia de la validez de constructo de esta versión del instrumento en la muestra mexicana.

La estructura factorial analizada para la medida del constructo de burnout académico corresponde a los planteamientos realizados por Maslach (1976) quien describe el burnout a través del agotamiento, el desinterés (cinismo) y sentimiento de incompetencia (inadecuación); esta estructura que ha sido replicada en diferentes investigaciones (Schaufeli, Salanova, GonzálezRoma, \& Bakker, 2002; Martínez \& Marques, 2005; Balsera, et al., 2016; Feitosa, Sousa, Alves, Gómez, \& Méndez, 2016; Weber \& Jaekel, 2000; De la Rosa, et al., 2015) coincide con la encontrada en esta investigación.

Específicamente al observar los índices de ajuste obtenidos por Boada y colaboradores (2015) en la versión traducida al castellano que fue la utilizada en el presente estudio, se concluye que el instrumento funciona adecuadamente en la muestra mexicana, mostrando una estructura trifactorial más diferenciada. Mientras que en el estudio con la muestra española, el factor "inadecuación" con dos reactivos, superpone gran parte de su varianza con el factor "cinismo", esto no ocurrió en la muestra mexicana, ya que se aprecia una clara diferencia entre estos factores al decrementar el valor de la correlación observada de $r=.92$ (Boada, et al., 2015) a r=.67.

En cuanto al AFC multi-grupo sobre el sexo de los encuestados, en primera instancia se observa que todos los pesos factoriales fueron aceptables $(\lambda<.30)$ siendo únicamente el reactivo 7 (Me obsesiono con temas relacionados con mi trabajo académico durante mi tiempo libre; del área del agotamiento) para el caso de los hombres, el que presenta un valor bajo, pero dentro del criterio planteado por Furr (2017). Por lo tanto, se acepta la invarianza factorial del modelo en hombres y mujeres a partir de los valores de chi cuadrado $\left(\mathrm{X}^{2}\right)$, índice de ajuste comparativo (CFI) y error de aproximación residual de la raíz de la media cuadrática (RMSEA) puesto que se alcanzaron los criterios planteados por Vanderberg y Lance (2000), Caycho (2017) y Cheung y Rensvold (2002); datos similares a los reportados por autores como Charry, Garzón, Pozo y Bretones (2018), quienes realizaron un análisis de este tipo en burnout académico con estudiantes hispanohablantes.

Como limitaciones del instrumento, si se adopta el modelo trifactorial sería recomendable reforzar la medida del constructo incluyendo nuevos items; ya que autores como Furr (2017), Lloret-Segura, Ferreres-Traver, Hernández-Baeza y Tomás-Marco (2014), y Ferrando y Anguiano (2010), refieren que para la correcta identificación de los factores es necesario que estén constituido al menos por 3 reactivos, y en este caso el factor inadecuación cuenta con solo dos ítems. Por otra parte, sería conveniente evaluar la estabilidad de 
la estructura factorial en otras muestras ya que en el presente estudio se ha trabajado solo con una muestra del centro del país y de una sola universidad, así como recoger evidencias en relación con otras variables. En consecuencia, para la investigación futura se realizan las siguientes propuestas: 1) la aplicación conjunta de la presente escala con otras a partir del establecimiento de una red nomológica para analizar los patrones particulares de asociación con otros atributos psicológicos, y 2) la realización de análisis homólogos al presente en muestras procedentes de otras regiones del país con la finalidad de comprobar la generalizabilidad del instrumento.

\section{Conclusiones}

En el presente estudio se muestran las propiedades psicométricas, estructura factorial e invarianza entre hombres y mujeres del SBI-U-9 (Boada, et al., 2015), mostrándose una adecuada bondad de ajuste al modelo trifactorial propuesto por Boada, et al. (2015) lo que supone una generalización que a su vez permite su aplicación en muestras mexicanas.

Finalmente, debido a los efectos del burnout académico, se considera indispensable contar con instrumentos como el presente, el cual permitirá dar cuenta objetivamente de la dimensión del fenómeno y proponer soluciones tanto a nivel institucional como individual.

\section{Referencias}

Aguilar-Bustamante, M., \& Riaño-Hernandez, D. (2013). Propiedades psicométricas del "School Burnout Inventory" SBI en población colombiana adolescente. Paper presented at the 34th Interamerican Congress of Psychology, 15-19 July, Brasilia (Brazil).

Balluerka, N., Gorostiaga, A., Alonso, I., \& Haramburu, M. (2007). La adaptación de instrumentos de medida de unas culturas a otras: una perspectiva práctica. Psicothema, 19(1), 124-133.

Balsera, G. M., Chavasco, G. G., López, G. R., Pérez, R. A., Sánchez, M. M., \& Villena, R. C. (2016). Estudio del Burnout en estudiantes de la Universidad de Granada: Influencia moduladora de variables de inteligencia emocional y clasificación por cursos, Monográfico de Psicología y Salud, 5, 27-32.

Barradas, A., M., Trujillo, C., P., Sánchez, B., A., \& López, G., J. (2017). Burnout estudiantil en universitarios veracruzanos. RIDE Revista Iberoamericana para la Investigación y el Desarrollo Educativo, 7(14), 1-19. doi:10.23913/ride.v7i14.267.

Barrera, R., Navarrom, A., \& Peris, M. (2015). El papel de la invarianza factorial en la validación del constructo calidad de servicio electrónico. Revista Europea de Dirección y Economía de la Empresa, 24(1), 13-24. doi:10.1016/j.redee.2014.07.001.

Boada, J., Merino, E., Sánchez, J. C., Prizmic, A. J., \& Vigil, A. (2015). Adaptation and psychometric properties of the SBI-U scale for Academic Burnout in university students. Anales de Psicología, 31(1), 290-297. doi:10.6018/analesps.31.1.168581.

Bollen, K. (1989). Structural equations with latent variables. New York: John Wiley and sons.

Caballero, C. (2012). El burnout académico: Prevalencia y factores asociados en estudiantes universitarios del área de la salud de la ciudad de Barranquilla. Doctoral dissertation. Universidad del Norte, Barranquilla (Colombia).

Caballero, C., Abello, L., Raymundo L., \& Palacios, S. (2007). Relationship between burnout, academic performance, and satisfaction concerning study, in college students. Avances en Psicología Latinoamericana, 25(2), 98-111.

Caballero, C., Bresó, E., \& González G. (2015). Burnout en estudiantes universitarios. Psicología desde el Caribe, 32(3), 424-441. doi:10.14482/psdc.32.3.6217.

Caballero, C., Hederich, C., \& Palacio, J. (2010). El burnout académico: Delimitación del síndrome y factores asociados con su aparición. Revista Latinoamericana de Psicología, 42(1), 131-146.

Caycho, T. (2017). Importancia del análisis de invarianza factorial en estudios comparativos en Ciencias de la Salud. Educación Médica Superior, 31(2), 1-3.

Charry, C., Garzón, A., Pozo, C., \& Bretones, B. (2018). Invarianza factorial del burnout 
académico para estudiantes hispanohablantes. Revista Mexicana de Investigación Educativa, 23(79), 1195-1215.

Cheung, W., \& Rensvold, R. B. (2009). Evaluating Goodnees-of-fit indexes for testing mesurement invariance. Structural Equation Modeling: A Multidisciplinary Journal, 9(2), 233-255.

doi:10.1207/S15328007SEM0902_5.

De la Rosa, G., Chang, S., Delgado, L., Oliveros, L., Murillo, D., Ortiz, R., ... \& Yhuri, N. (2015). Level of stress and coping strategy in medical students compared with students of other careers, PubMed, 151(1), 443-449.

Domínguez, S. A., \& Merino, C. (2019). Medición con Ítem Único del Agotamiento Emocional Académico en Estudiantes Universitarios Peruanos: Evidencias de Validez y Confiabilidad. Revista Iberoamericana de Diagnóstico y Evaluación - e Avaliação Psicológica. 50(1), 45-56. doi:10.21865/RIDEP50.1.04.

Elousa, P. (2005). Evaluación progresiva de la invarianza factorial entre las versiones original y adaptada de una escala de autoconcepto. Psicothema, 17(2), 356-362.

Fabrigar, L. R., Wegener, D. T., MacCallum, R. C., \& Strahan, E. J. (1999). Evaluating the use of exploratory factor analysis in psychological research. Psychological Methods, 4(3), 272299. doi:1082-989X/99/S3.00.

Feitosa-Sousa, K., Sousa-Lima, A., AlvesFernandes, S., Gomes-Carcalho, P., \& MendesAraújo, L. (2016). Síndrome de agotamiento laboral (burnout) y sus representaciones entre profesionales de la salud. Invest Enferm. imagen desarr, 18(2),137-152.

doi:10.11144/Javeriana.ie18-2.sbpe.

Ferrando, P. J., \& Lorenzo-Seva, U. (2017). Program FACTOR at 10: Origins, development and future directions. Psicothema, 29(2), 236-241. doi: $10.7334 /$ psicothema2016.304

Finney, S. J., \& DiStefano, C. (2006). Nonnormal and categorical data in structural equation modeling. In G. R. Hancock \& R. O. Mueller (Eds.), Structural equation modeling: A second course (pp. 269-314). Greenwich, CT: Information Age.
Fiorilli, C., Galimberti, V., De Stasio, S., Di Chiacchio, C., \& Albanese, O. (2014). L'utilizzazione dello School Burnout Inventory (SBI) con studenti italiani di scuola superiore di primo e secondo grado. Psicologia dello Sviluppo, 13(3), 403-424. doi:10.1449/78365

Freudenberger, H. J. (1974). Staff burn-out. Journal of social issues, 30, 159-165. doi:10.1111/j.1540-4560.1974.tb00706.x

Furr, M. (2017). Psychometrics: An introduction. SAGE Publications: EUA.

Hederich, M. C., \& Caballero, D.C. (2016). Validación del cuestionario Maslach Burnout Inventory-Student Survey (MBI-SS) en contexto académico colombiano. Revista CES Psicología, 9(1), I-XV.

Herrera, L., Mohamed, L., \& Cepero, S. (2016). Cansancio Emocional en estudiantes universitarios. Revista de Educaçăo e Humanidades, 9, 173-191.

Hu, L. T., \& Bentler, P. M. (1999). Cutoff criteria for fit indexes in covariance structure analysis: Conventional criteria versus new alternatives. Struct Equ Modeling, 6,1-55. doi:10.1080/10705519909540118.

Lloret-Segura, S., Ferreres-Traver, A., Hernández-Baeza, A., \& Tomás-Marco, I. (2014). El análisis factorial exploratorio de los ítems: Una guía práctica, revisada y actualizada. Anales de Psicología, 30(3), 11511169. doi:10.6018/analesps.30.3.199361.

Manzano, P. A., \& Zamora, M. S. (2009). Sistema de Ecuaciones Estructurales: Una herramienta de investigación. Cuaderno Técnico 4. CENEVAL: México.

Martínez, I., \& Marques, A. (2005). Burnout en estudiantes universitarios de España y Portugal y su relación con variables académicas. Aletheia, (21), 21-30.

Maslach, C. (1976). Burned-out. Human behavior, $5,16-22$.

Maslach, C., \& Jackson, S. E. (1981). Maslach Burnout Inventory. Research Edition. Palo Alto, CA: Consulting Psychologist Press.

Maslach, C., \& Jackson, S. E. (1982). Burnout in health professions: A social psychological analysis. En G. Sanders y J. Suls, (eds), Social Psychology of health and illness. Hillsdale, N.J.:Erlbaum. 
Maslach, C., \& Schaufeli, B. (1993) Historical and conceptual developments of burnout. En W.B. Schaufeli,; Ch, Maslach y T. Marek (Eds). Professional Burnout. London: Taylor and Francis.

Maslach, C., Jackson, S. E., \& Leiter, M. P. (1996). Maslach Burnout Inventory. Third Edition. Palo Alto,CA: Consulting Psychologist Press.

Merino, C., Delgadillo, A., \& Caballero, R. (2013). ¿Burnout en adolescentes?: Validez estructural del inventario de burnout escolar (SBI) (Burnout in adolescents: Structural validity School Burnout Inventory (SBI)). Paper presented at the 34th Interamerican Congress of Psychology, 15-19 July, Brasilia (Brazil).

Montiel, P. A., \& Chong, B. M. C., (2019). La práctica de la evaluación del aprendizaje como generadora del Síndrome de Burnout Académico en los estudiantes de la Licenciatura en Ciencias de la Educación. Revista Metropolitana de Ciencias Aplicadas, 2(1), 71-77.

Moreno, J. B., Rodríguez, C. R., \& Escobar R. E. (2001). La evaluación del burnout profesional factorialización del MBI-GS. Un análisis preliminar. Ansiedad y Estrés, 7(1), 69-78.

Moyano, N., \& Riaño-Hernández, D. (2013). Burnout escolar en adolescentes españoles: Adaptación y validación del School Burnout Inventory. Ansiedad y Estrés, 19, 95-103.

Ochoa M. G., Musi L. B., Olivas Á. J., \& Espinosa, R., (2018). Validez de contenido del School Burnout Inventory para adolescentes escolarizados mexicanos. Ponencia presentada en las XXVI Congreso Mexicano de Psicología, 3, 4 y 5 de octubre Ciudad Juárez, Chihuahua, México.

Oliva. E., Andrade, S., Rodríguez, A., Vieira, E., \& Matos, T. (2012). Burnout Syndrome and associated factors among medical students: A cross-sectional study. Clinics, 67(6), 573-579. doi:10.6061/clinics/2012(06)05

Osorio, G. M., Parrello, S., \& Prado, R. C. (en prensa). Burnout académico en una muestra de estudiantes. Enseñanza e Investigación en Psicología, 2(1).

Palacio-Sañudo, J., Caballero-Domínguez, C., González-Gutiérrez, O., Gravini-Gómez, M.,
\& Contreras-Santos, K. (2012). Relación del burnout y las estrategias de afrontamiento con el promedio académico en estudiantes universitarios. Universitas Psychologica,11(2), 535-544.

Parrello, S., Ambrosetti, A., Iorio, I., \& Castelli, L. (2019). School Burnout, Relational, and Organizational Factors. Front. Psychol. 10, 16. doi:10.3389/fpsyg.2019.01695.

Pedrosa, I., \& García, E. (2016). Síndrome de Burnout en Árbitros de Élite: La Liga de Fútbol Profesional Española (LFP) a Estudio. Revista Iberoamericana de Diagnóstico y Evaluación - e Avaliação Psicológica. 42(2), 59-68. doi:10.21865/RIDEP42_59.

Rosales, Y., \& Rosales, F. (2013). Burnout estudiantil universitario: Conceptualización y estudio. Salud mental, 36(4), 10-22.

Salmela-Aro, K., \& Kuntu, K. (2010). Study Burnout and Engagement in Higher Education. Unterrichtswissenschaft, 318- 333.

Salmela-Aro, K., Kiuru, N., Leskinen, E., \& Nurmi, J. E. (2009). School-Burnout Inventory (SBI) - Reliability and validity. European Journal of Psychological Assessment, 25, 48-57. doi:10.1027/1015-5759.25.1.48.

Schaufeli, W. B., Salanova, M., González-Romá, V., \& Bakker, A. (2002). The measurement of burnout and engagement: A Two Sample Confirmatory Factor Analytic Approach. Journal of Happiness Studies, 3, 71-92. doi:10.1023/A:1015630930326.

Thompson, B., \& Daniel, L. G. (1996). Factor analytic evidence for the construct validity of scores: A historical overview and some guidelines. Educational and Psychological Measurement, 56, 197-208. doi:10.1177/0013164496056002001.

Vanderberg, R., \& Lance, C. (2000). A review and synthesis of the mesurement invriance literature: Suggestions, practices, and recomendatios for organizational research. Organizational Research Methods, 3(1), 4-69. doi:10.1177/109442810031002.

Weber, A., \& Jaekel, A. (2000). Burnout syndrome: A disease of modern societies?. Occup. $\quad$ Med, 50(7), 512-517. doi:10.1093/occmed/50.7.512. 


\section{Anexo}

School Burnout Inventary para estudiantes universitarios (SBI-U-9) versión validada al castellano por Boada, et al., (2015)

Instrucciones. Por favor, elige la alternativa que mejor describe tu situación (estimación del mes pasado) en cuanto a tu situación en la Universidad.

\begin{tabular}{|l|l|l|l|l|l|}
\hline $\begin{array}{l}\text { 1 Totalmente } \\
\text { en desacuerdo }\end{array}$ & $\begin{array}{l}\text { 2 Relativamente } \\
\text { en desacuerdo }\end{array}$ & 3 En desacuerdo & $\begin{array}{l}4 \text { Relativamente } \\
\text { de acuerdo }\end{array}$ & 5 De acuerdo & $\begin{array}{l}6 \text { Totalmente de } \\
\text { acuerdo }\end{array}$ \\
\hline
\end{tabular}

1.-Me siento agobiado/a por el trabajo académico (AGO 1).

2.-Siento una falta de motivación en mis actividades universitarias, y a menudo, pienso en dejarlo (CIN 1).

3.-A menudo tengo una sensación de insuficiencia en mis actividades universitarias (INAD 1).

4.-A menudo me cuesta dormir por temas relacionados con mi trabajo académico (AGO 2).

5.-Siento que estoy perdiendo interés en mi trabajo académico (CIN 2).

6.- Me pregunto constantemente si mi trabajo académico tiene algún significado (CIN 3).

7.-Me obsesiono con temas relacionados con mi trabajo académico durante mi tiempo libre (AGO 3).

8.-Solía tener unas expectativas más altas de mi trabajo académico que las que tengo ahora (INAD 2).

9.-La presión de mi trabajo universitario me causa problemas en mis relaciones personales con los demás (AGO 4).

Nota. $\mathrm{AGO}=$ Agotamiento del trabajo universitario; $\mathrm{CIN}=$ Cinismo hacia el sentido universitario; INAD=Sentido de inadecuación a la universidad 